\title{
Asymptotic dependence of Gross-Tulub polaron ground-state energy in the strong coupling region
}

\author{
N.I. Kashirina \\ V. Lashkaryov Institute of Semiconductor Physics, NAS of Ukraine \\ 41, prospect Nauky, 03028 Kyiv, Ukraine \\ E-mail: kashirina@isp.kiev.ua
}

\begin{abstract}
The properties of translationally invariant polaron functional have been investigated in the region of strong and extremely strong coupling. It has been shown that the Gross-Tulub polaron functional obtained earlier using the methods of field theory was derived only for the region $\alpha \leq 10$, where $\alpha$ is the Fröhlich constant of the electron-phonon coupling. Various representations of exact and approximate polaron functionals have been considered. Asymptotic dependences of the polaron energy have been obtained using a functional extending the Gross-Tulub functional to the region of extremely strong coupling. The asymptotic dependence of polaron energies for an extremely strong coupling are $E_{p} \approx-0.31683 \alpha^{4 / 3}$ (for the one-parameter variational function $f_{k}$ ), and $E_{p} \approx-0.31767 \alpha^{4 / 3}$ (for a two-parameter function $f_{k}^{\prime}$ ). It has been shown that the virial theorem 1:3:4 holds for the two-parameter function $f_{k}^{\prime}$. Minimization of the approximate functional obtained by expanding the exact GrossTulub functional in a series on $1 / \alpha$ leads to a quadratic dependence of the polaron energy. This approximation is justified for $\alpha \approx 7.5 \ldots 8$. For a two-parameter function $f_{k}^{\prime}$, the corresponding dependence has the form $E_{p} \approx-0.125 \alpha^{2}$. However, the use of approximate functionals, in contrast to the strict variational procedure, when the exact polaron functional varies, does not guarantee obtaining the upper limit for the polaron energy.
\end{abstract}

Keywords: polaron, Fröhlich Hamiltonian, electron-phonon interaction.

Manuscript received 11.09.17; revised version received 13.11.17; accepted for publication 07.12.17; published online 07.12.17.

\section{Introduction}

The basis of polaron theory was laid down by Pekar [1]. A brief historical note on origins of polaron physics is given by Dykman and Rashba [2]. The first works in this direction were devoted to a strong coupling polaron. Despite the fact that variation wave function of the strong-coupling polaron (Pekar polaron) does not possess translational invariance, the strong-coupling polaron energy, founded by the variational method, is the upper limit of the continual polaron energy. A con- siderable amount of works was devoted to the presentation of methods of the intermediate coupling in the polaron theory, including reviews and monographs $[3,4]$. Using the Feynman variational method [5], it was possible to find the lowest values of the polaron energy for the entire range of the electron-phonon coupling constant, with the exception of the region of extremely strong coupling, where the Pekar polaron energy was lower. The table of values of the polaron energy obtained by various variational methods is given in the monograph [4, p. 29]. 
In the works of Gross and Tulub [6-8], the field theory method was used for finding the energy of the translationally invariant polaron. This approach generalized the method of the intermediate coupling proposed by Lee Lowe and Pines [9]. As was pointed out in $[8,10]$, for a continual polaron the region of existence is bounded on the electron-phonon coupling parameter by the values $\alpha \sim 7.5 \ldots 8$, where $\alpha$ is the dimensionless Fröhlich constant given by:

$$
\alpha=g^{2}=\frac{1}{2}\left(\frac{1}{\varepsilon_{\infty}}-\frac{1}{\varepsilon_{0}}\right) \frac{e^{2}}{\hbar \omega_{k}^{0} l_{0}}, l_{0}=\sqrt{\frac{\hbar}{2 m^{*} \omega_{k}^{0}}},
$$

$\varepsilon_{0}$ and $\varepsilon_{\infty}$ are static and high-frequency permittivities, respectively, $\omega_{k}^{0}$ is the energy of longitudinal optical phonons, $l_{0}-$ effective Bohr radius, $m^{*}-$ effective electron mass.

The restriction of the phonon spectrum by the limiting wave vector $k_{m}$ also leads to a constraint on the value of the electron-phonon interaction by the constant $\alpha_{m} \sim 8 \ldots 10[8,10]$. From this point of view, investigation of the asymptotic dependences of the polaron energy at $\alpha \rightarrow \infty$ is an important, but rather a mathematical, problem, since the region of crystal parameters for which $\alpha>>10$ lies beyond the boundaries of the existence of a real (physical) continual polaron. As shown in $[11,12]$, the Tulub polaron functional [8] can be used to find the functional of the translation-invariant bipolaron. The discussion material devoted to the consideration of the asymptotic dependences of the polaron and bipolaron energy, which were found by the Tulub method [8], is described in the works [12-16].

The simple form of the exact functional of translationally invariant polaron was found in [17]. That makes it possible to extend the region of admissible $\alpha$ values for the Gross-Tuloub polaron $[6,7]$ to the strongcoupling region. This paper is devoted to investigation of the asymptotic dependence of the polaron energy in the region of the extremely strong coupling. The polaron energy is determined by minimizing the polaron functional obtained in [17].

\section{Exact and approximate functionals in the polaron theory}

Polaron functionals obtained using various theoretical methods can have a rather complex form. In the similar cases, approximate methods are used to simplify the resulting functional. The expansion of the polaron functional into a series on the inverse Fröhlich coupling constant $1 / \alpha<<1$ with subsequent minimization on the variational parameters is one of the examples of obtaining an approximate value of the polaron energy for the intermediate and strong coupling region $[8,10,18]$.
Variation of approximate functionals does not guarantee that an upper limit of the polaron energy can be found. Thus, variation of the approximate functional obtained by Feynman in the strong-coupling region was accompanied by the following comment on the magnitude of the polaron energy $E$ : "The approximations do not keep $E$ as an upper limit as, unfortunately, the further terms, of order $1 / \alpha^{2}$ are probably positive". In the general case, approximate methods can give reasonable values of the polaron energy not for the entire range of variational parameters. In this relation, it is necessary to find the region of admissible values of the variational parameters, for which the functionals obtained using the approximate methods are valid.

From the standpoint of the comparing approximate and exact methods, one of the most interesting objects is the translationally-invariant (TI) polaron functionals obtained in the works by Gross [6] and Tulub [7].

Up to the notation, the polaron functional obtained using the field theory methods in the papers of Gross and Tulub $[6,7]$ has the form:

$$
\begin{aligned}
& J_{\mathrm{GT}}=\Delta J_{\mathrm{GT}}+2 g \sum_{k} V_{k} f_{k}+\sum_{k} \omega_{k}^{0} f_{k}^{2}, \\
& \Delta J_{\mathrm{GT}}=\frac{3}{4 \pi} \int_{1}^{\infty} \frac{d s}{\sqrt{s}} \operatorname{arctg}[\operatorname{Im} D(s) / \operatorname{Re} D(s)]= \\
& =\frac{3}{2 \pi} \int_{0}^{\infty} d k \cdot k \cdot \operatorname{arctg}\left[\operatorname{Im} D\left(\omega_{k}^{2}\right) / \operatorname{Re} D\left(\omega_{k}^{2}\right)\right], \\
& D(s)=1+Q(s)=1+\frac{1}{3 \pi^{2}} \int_{0}^{\infty} \frac{p^{4} f_{p}^{2} \omega_{p}}{\omega_{p}^{2}-s} d p,
\end{aligned}
$$

where $V_{k}=k^{-1} \sqrt{4 \pi \alpha l_{0}}, \omega_{k}=\omega_{k}^{0}+k^{2} / 2$, (hereinafter $\left.\omega_{k}^{0}=1\right), f_{k}$ is the variational function of the GrossTulub method. The function $D(s)$ coincides in its form with the dynamic susceptibility of a degenerate electron gas.

We write expression (2) in an equivalent form, which is more convenient for variational calculations:

$$
\begin{aligned}
& \Delta J_{\mathrm{GT}}=\frac{3}{4 \pi} \int_{1}^{\infty} \frac{d s}{\sqrt{s}} \arcsin \{\operatorname{Im} D(s) /|D(s)|\}= \\
& =\frac{3}{2 \pi} \int_{0}^{\infty} d k \cdot k \cdot \arcsin \left\{\operatorname{Im} D\left(\omega_{k}^{2}\right) /\left|D\left(\omega_{k}^{2}\right)\right|\right\} .
\end{aligned}
$$

As was shown in [17], the functional (1) was written in $[6,7]$ only for the case when the complex quantity $D(s)$ is located in the first quarter of the 
complex plane, although the authors of $[6,7]$ do not stipulated this particularity.

In [8] $f_{k}$ minimized the polaron functional in the strong-coupling region was chosen in the form of oneparameter Gaussian function:

$$
f_{k}=-V_{k} e^{-k^{2} / 2 a^{2}}
$$

where $V_{k}=k^{-1} \sqrt{4 \pi \alpha l_{0}}, a$ is the variational parameter.

We draw attention to the fact that the function (5) was chosen in a form that coincides with the Fourier component of the normalized Gaussian electron function of the Pekar polaron in the strong coupling limit. For the variational function defined by the expression (5), the function $D(s)$ was obtained in [8] after substituting (5) into (3):

$$
\begin{aligned}
& \operatorname{Re} D\left(\omega_{k}^{2}\right)=1+\lambda v(y)=\lambda(\mu+v(y)), \\
& \operatorname{Im} D\left(\omega_{k}^{2}\right)=\frac{k^{3} f_{k}^{2}}{6 \pi}, \\
& v(y)=1-y e^{-y^{2}} \int_{0}^{y} e^{t^{2}} d t-\xi e^{\xi^{2}} \int_{\xi}^{\infty} e^{-t^{2}} d t,
\end{aligned}
$$

where $\quad \lambda=4 g^{2} a / 3 \sqrt{2 \pi}, \quad \mu=1 / \lambda, \quad y=k / a$, $\xi=\sqrt{y^{2}+4 / a^{2}}$.

For $y>>1$, the asymptotic expansion $v(y) \approx 1-3 \lambda / 4 y^{4}$ is valid. For $y=y_{m}=(3 \lambda / 4)^{1 / 4}$, $v\left(y_{m}\right)=0$.

For the two-parameter function:

$$
f_{k}^{\prime}=-N V_{k} e^{-k^{2} / 2 a^{2}}
$$

where $a$ and $N$ are the variational parameters, we make substitution $\lambda \rightarrow \lambda^{\prime}=4 g^{2} N^{2} a / 3 \sqrt{2 \pi}$ in Eq. (6).

Tulub [8] obtained a polaron functional in the form convenient for investigating not only the intermediate coupling, but also the region of extremely strong coupling. For the recoil terms in [8] the following expression was obtained:

$$
\begin{aligned}
& \Delta J_{T}=\frac{1}{4 \pi^{2}} \int_{0}^{\infty} \frac{k^{4} f_{k}^{2} d k}{(1+Q(1))}+ \\
& +\frac{1}{12 \pi^{4}} \int_{0}^{\infty} \int_{0}^{\infty} \frac{k^{4} f_{k}^{2} p^{4} f_{p}^{2} \omega_{p}\left\{\omega_{k} \omega_{p}+\omega_{k}\left(\omega_{k}+\omega_{p}\right)+1\right\}}{\left(\omega_{k}+\omega_{p}\right)^{2}\left(\omega_{p}^{2}-1\right)\left|D\left(\omega_{p}^{2}\right)\right|^{2}} d p d k .
\end{aligned}
$$

In what follows, Eq. (1) with the recoil term determined by Eqs. (2) and (3) will be called the GrossTulub functional, and the Tulub functional is the expression (1), in which the first term is written in the form (9):

$$
J_{\mathrm{T}}=\Delta J_{\mathrm{T}}+2 g \sum_{k} V_{k} f_{k}+\sum_{k} \omega_{k}^{0} f_{k}^{2} .
$$

The Tulub functional (10) can be represented in an approximate form. The variational parameter $a>>1$ for the strong-coupling limit. In this case, in Eq. (9), we can approximately put $\omega_{k} \approx k^{2} / 2$. After simplifications for the two-parameter function $f_{k}^{\prime}$, we obtain an approximate form for the functional of the translationally invariant polaron:

$$
\begin{aligned}
& J_{\mathrm{T}} \approx J_{\mathrm{T}}^{\prime}=\frac{3}{16} a^{2}\left[1+q\left(\frac{1}{\lambda^{\prime}}\right)\right]-\frac{g^{2} a N}{\sqrt{\pi}}\left(2-\frac{N}{\sqrt{2}}\right), \\
& q\left(\frac{1}{\lambda^{\prime}}\right)=\frac{2}{\sqrt{\pi}} \int_{0}^{\infty} \frac{e^{-y^{2}}(1-\Omega(y))}{[(1 / \lambda)+v(y)]^{2}+\pi y^{2} e^{-2 y^{2}} / 4} d y, \\
& \Omega(y)=2 y^{2}\left\{\left(1+2 y^{2}\right) y e^{y^{2}} \int_{y}^{\infty} e^{-t^{2}} d t-y^{2}\right\} .
\end{aligned}
$$

Further simplification is related with calculation of the integral (12). Putting in (12) $q\left(\frac{1}{\lambda^{\prime}}\right) \approx q(0) \approx 5.75$, we obtain the following simplified expression:

$$
J_{\mathrm{T}} \approx J_{\mathrm{T}}^{\prime \prime}=\frac{3}{16} a^{2}[1+5.75]-\frac{g^{2} a N}{\sqrt{\pi}}\left(2-\frac{N}{\sqrt{2}}\right)
$$

Eq. (11) allows us to investigate the asymptotic behavior of the functional (10) in the limit $\alpha \rightarrow \infty$, at the same time, Eq. (13) is valid only for continuum polaron. This region is bounded by the coupling constant $\alpha \approx 8 \ldots 10$. In the range of $\alpha \leq 10$, the value $\operatorname{Re} D\left(\omega_{k}^{2}\right)>0$.

Klimin and Devreese [13] showed that the deltalike singularity appearing near the second zero of the expression $\operatorname{Re} D\left(\omega_{k}^{2}\right)=1+\lambda v(y)$ must be taken into account in the integral (12). In this case, instead of the approximate expression (13), we obtain:

$$
\begin{aligned}
& J_{\mathrm{T}}^{a s}=\frac{3}{16} a^{2}[1+5.75]+\delta E_{m}(a, \alpha)-\frac{2 \alpha N a}{\sqrt{\pi}}+\frac{\alpha N^{2} a}{\sqrt{2 \pi}} \\
& \delta E_{m}=\frac{3}{8} a^{2} \sqrt{3 \lambda^{\prime}}=\frac{3}{2}\left(\frac{N^{2} \sqrt{2}}{8 \sqrt{\pi}} \alpha a^{5}\right)^{1 / 2} .
\end{aligned}
$$


In contrast to [13], we recorded the expression (14) for the two-parameter function $f_{k}^{\prime}$ defined by Eq. (8), since it was shown in [19] that the one-parameter function $f_{k}(5)$ is not enough flexible to satisfy the virial relations of the polaron theory [1].

An approximate method of expanding the integrand of Eq. (12) with respect to $1 / \lambda$, which was used in [8], results in a quadratic dependence of the leading term on $\alpha$ in the expansion of the polaron energy. Thus, the oneparameter test function $f_{k}$ was chosen in [8]. For $1 / \lambda \rightarrow 0$ in (12), the polaron energy is $E_{p} \approx-0.105 \alpha^{2}$.

This energy is extremely close to the value of Pekar polaron energy $E_{P} \approx-0.106 \alpha^{2}$ in the strong coupling limit [1].

One of the differences between the BuimistrovPekar TI polaron functional [18] and the TI functionals obtained by Gross [6] and Tulub $[7,8,10]$ is that the strong coupling term, which leads to a quadratic dependence of the energy on $\alpha$, can be distinguished from the Buimistrov-Pekar functional without expansion on $1 / \alpha$. The functionals (1) and (10) does not allow us to isolate a part of the strong coupling in the general form. In this case, it can turn out that quadratic dependence characterizing a strong coupling will prove to be a good approximation only for a certain range $\alpha$, but not for all $\alpha>>1$.

In [8], it was noted that the strong-coupling region, for which the approximation (13) is valid, actually reduces to zero, since electron-phonon interaction constant $\alpha \leq \alpha_{m} \approx 8$ for real crystals. Any singularities did not arise for such $\alpha$ in the integrand of Eq. (12), so expansion on $1 / \lambda$ near $\alpha \approx 8$ could be regarded as a good approximation of the exact functional. The approximate energy of the Tulub polaron can be found after varying (13) on $a$. For the two-parameter function $f_{k}^{\prime}$, the corresponding energy is $E_{p s}^{\prime \prime} \approx-0.125 \alpha^{2}$, which is located below the exact numerical value $E_{\mathrm{M}}=-0.1085128 \alpha^{2}$ obtained by Miyake [20] for the strong-coupling limit. However, $E_{p s}^{\prime \prime}$ was obtained as a result of varying the approximate functional (13), therefore it is not the upper limit for polaron energy. In addition, it is necessary to determine the range of admissible values on $\alpha$, for which the approximate functional (13) does not lead to understated polaron energy, since instead of a strict variational procedure, we used an approximate method.

Not only the approximate functional can lead to understated polaron energy. Variation of the exact functional without checking the results of calculations for self-consistency with the range of admissible values of the initial functional can also lead to unreasonably low values of the polaron energy. For example, we vary the Gross-Tulub functional (1) determined, as was shown in [17], only in the first coordinate quarter of the complex plane. Taking (4) into account, we obtain:

$$
J_{\mathrm{GT}}=\frac{3 a^{2}}{2 \pi} \int_{0}^{\infty} d y \cdot Z(y)+2 g \sum_{k} V_{k} f_{k}+\sum_{k} \omega_{k}^{0} f_{k}^{2}
$$

$$
\begin{aligned}
& Z(y)=y \operatorname{At}(y), \quad \operatorname{At}(y)=\arcsin \left\{\operatorname{Im} D\left(\omega_{y}^{2}\right) /\left|D\left(\omega_{y}^{2}\right)\right|\right\}, \\
& y=k / a .
\end{aligned}
$$

We compare the results of minimization (15) with the energy obtained by minimizing the same functional but determined for the entire upper half-plane. According to [17], the generalized functional has the form:

$$
\widetilde{J}_{\mathrm{GT}}=\frac{3 a^{2}}{2 \pi} \int_{0}^{\infty} d y \cdot \widetilde{Z}(y)+2 g \sum_{k} V_{k} f_{k}+\sum_{k} \omega_{k}^{0} f_{k}^{2},
$$

where $\widetilde{Z}(y)=y \cdot \widetilde{A} t(y)$;

$$
\tilde{A} t(y)=\left\{\begin{array}{lll}
A t(y), & \text { if } & \operatorname{Re} D\left(\omega_{y}^{2}\right) \geq 0 \\
\pi-A t(y), & \text { if } & \operatorname{Re} D\left(\omega_{y}^{2}\right)<0
\end{array}\right\} .
$$

As was shown in [17], the functionals (10) and (17) are equivalent in the region $\alpha \leq 26$. In the region $\alpha>26$, it is necessary to investigate asymptotic dependences, which are valid for large $\alpha$ values and the integration variable $y$. Numerical calculations according to exact formulas are complicated in relation with the appearance of extremely small values of the exponential function $\exp \left(-2 y^{2} / a^{2}\right)$ for $y / a>>1$.

Fig. 1a shows the dependence of the Gross-Tulub polaron energy $\widetilde{E}_{\mathrm{GT}}$ on $\alpha$ obtained by minimizing the exact functional (17). Minimization using the functional (10) leads to the same results (coincidence is with 6 significant digits). $E_{p s}^{\prime \prime}$ - polaron energy obtained using the functional (13). Energy $E_{\mathrm{GT}}$ obtained by minimization of the functional (15) begins to fall down from $\alpha \approx 10$ approaching a quadratic dependence and leads to understated values of the polaron energy. For very large $\alpha$, it leads to an asymptotics $E_{\mathrm{GT}}^{\prime} \approx-0.135 \alpha^{2}$. In the same figure, the corresponding dependence for the Feynman polaron energy $E_{\mathrm{F}}[9]$ are given for comparison. The functionals (13), (15) and (17) were minimized using the two-parameter variational function $f_{k}^{\prime}$. We use term the "exact functional" to distinguish a functional, minimization of which provides the upper bound of the polaron energy, and an approximate functional that does not guarantee this. 


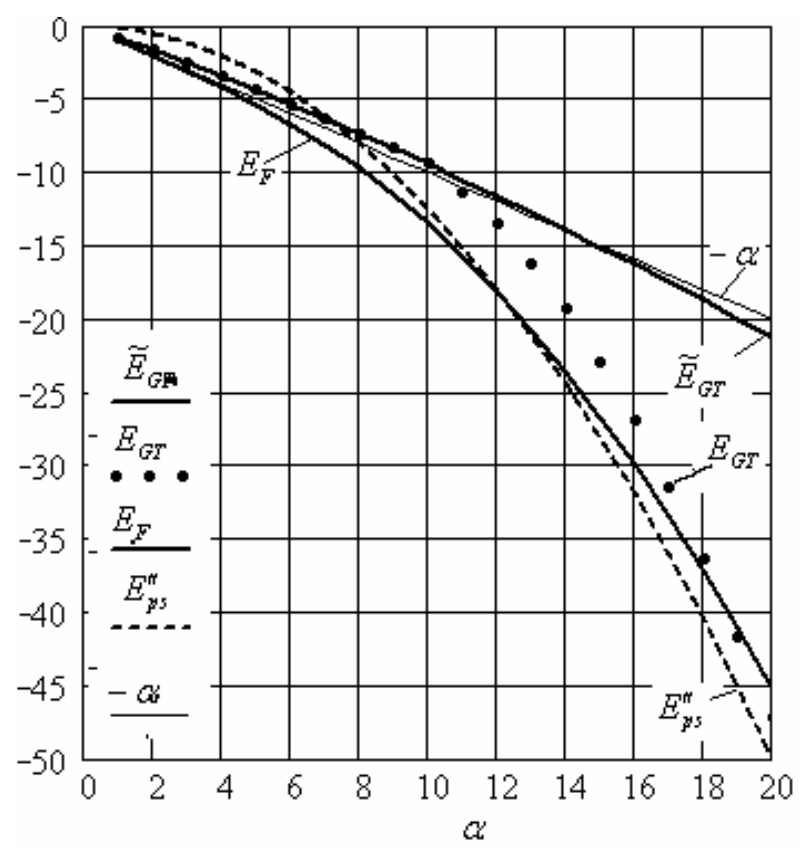

Fig. 1a. Dependences of polaron energy on $\alpha$ for various functionals. $E_{p s}^{\prime \prime}, E_{\mathrm{GT}}$ and $\widetilde{E}_{\mathrm{GT}}$ are the energies obtained by minimization of the functionals (13), (15) and (17), respectively; $E_{\mathrm{F}}$ is the Feynman polaron energy.

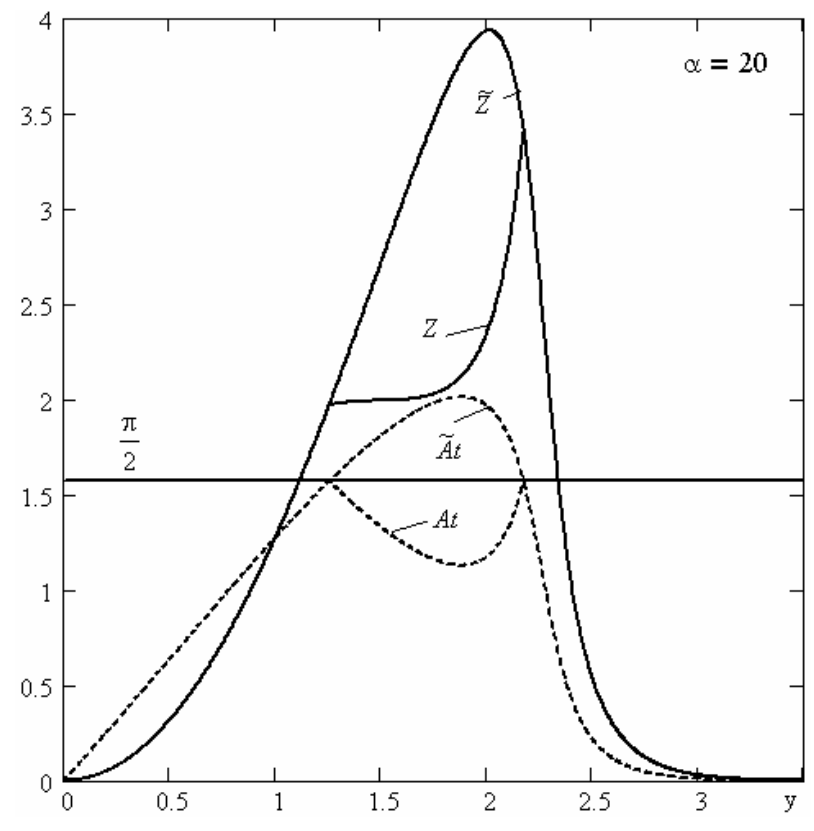

Fig. 1b. The integrands of the recoil terms in the functionals (15) and (17). The factor $3 a^{2} / 2 \pi$ is omitted.

Fig. 1b shows the integrands of the recoil terms in expressions (15) and (17), as well as the values of $A t(y)$ and $\tilde{A} t(y)$. Comparison of $Z(y)$ and $\widetilde{Z}(y), A t(y)$ and $\tilde{A} t(y)$ clearly demonstrates the reason for the understatement of the energy obtained using the functional (15), which is valid only for $D(s)$ located in the first quarter of the complex coordinate plane, when $\operatorname{Re} D(s) \geq 0$. As soon as there are areas for which $\operatorname{Re} D(s)<0$, the integrand starts to deviate downward from the correct value. Minimization of the functional (17) leads to correct results, which completely coincides with the polaron energy obtained using the Tulub functional (10).

\section{Investigation of the asymptotic dependence of the strong-coupling polaron}

Let us pass to definition of asymptotics on $\alpha$ of energy obtained using the exact functional (17) in the strongcoupling limit. For $1 / \lambda=0, D\left(\omega_{k}^{2}\right)$ has a unique zero on the real axis, coinciding with the zero of the function $v(y)$ defined by Eq. (7). Therefore, $\operatorname{Re} D\left(\omega_{k}^{2}\right)$ tends asymptotically to zero from the sides of the negative values, and the integrand $\widetilde{Z}(y)=y \widetilde{A} t(y) \rightarrow \infty$ in accordance with Eq. (18). I.e., the positive contribution to the phonon functional increases indefinitely, and the polaron energy tends to zero. Thus, expansion in a series on the parameter $1 / \lambda<<1$ is not justified in this case. It is necessary to save in $D\left(\omega_{k}^{2}\right)$ the small but finite parameter $1 / \lambda$. It provides the returning $D\left(\omega_{k}^{2}\right)$ from the second quarter of the complex plane to the first, where $\operatorname{Re} D\left(\omega_{k}^{2}\right) \geq 0$. When $1 / \lambda<<1$ and $y>>1$, the right decreasing part of the function $\tilde{Z}(y)$ in Fig. $1 \mathrm{~b}$ takes the vertical position and the integrand function can be approximated using the theta function $\tilde{Z}(y)=y \tilde{A} t(y) \theta\left(y-y_{m}\right)$ :

$$
\begin{aligned}
& \frac{3 a^{2}}{2 \pi} \int_{0}^{\infty} d y \cdot \widetilde{Z}(y) \mid(\text { for } 1 / \lambda<<1) \approx \\
& \left.\approx \frac{3 a^{2}}{2 \pi} \int_{0}^{\infty} d y \cdot \theta\left(y-y_{m}\right)\{\tilde{Z}(y)\}\right|_{1 / \lambda=0}= \\
& =\left.\frac{3 a^{2}}{2 \pi} \int_{0}^{y_{m}} d y \cdot\{\tilde{Z}(y)\}\right|_{1 / \lambda=0},
\end{aligned}
$$

where $\theta\left(y-y_{m}\right)=\left\{\begin{array}{ccc}1 & \text { for } & y \leq y_{m} \\ 0 & \text { for } & y>y_{m}\end{array}\right\}$,

$y_{m}=\operatorname{root}\left\{\operatorname{Re} D\left(\omega_{k}^{2}\right)\right\}$.

The polaron energy for extremely large $\alpha$ $(1 / \lambda<<1)$ can be obtained by minimizing the functional: 
$\tilde{J}_{\mathrm{GT}}=\left.\frac{3 a^{2}}{2 \pi} \int_{0}^{y_{m}} d y \cdot\{\tilde{Z}(y)\}\right|_{1 / \lambda=0}+2 g \sum_{k} V_{k} f_{k}+\sum_{k} \omega_{k}^{0} f_{k}^{2}$

where $y_{m}=[3 \lambda(a, \alpha, N) / 4]^{1 / 4}$ is the asymptotic value of $\operatorname{root}\left\{\operatorname{Re} D\left(\omega_{y}^{2}\right)\right\}$ for $y=k / a>>1, a, N$ are the variational parameters.

For one-parameter function $f_{k}$, the asymptotic dependence of the polaron energy defined by minimization of the functional (20) has the form: $E_{\mathrm{GT}}^{a s}=\left.\min _{a}\left\{J_{\mathrm{GT}}\right\}\right|_{\alpha>>1}=-0.31683 \alpha^{4 / 3}$. This asymptotic behavior coincides with the asymptotics obtained for the Tulub functional (11) in [13] by varying the approximate functional (14). The approximate functional (14) was obtained by expansion of the Tulub functional (11) on $1 / \lambda$, but taking into account the additional term associated with the appearance of a singularity in the integrand in Eq. (12) appearing for small but finite values $1 / \lambda$ at the point $y=y_{m}$.

For the two-parameter function $f_{k}^{\prime}$, the corresponding asymptotic dependence for the functional $\widetilde{J}_{\mathrm{GT}}$ defined by Eq. (20) has the form:

$\widetilde{E}_{\mathrm{GT}}^{a s}=-0.31767 \alpha^{4 / 3}$.

\section{The strong-coupling limit and the virial theorem}

We note that the variation of the approximate functionals (11), (13) and (14) with using the one-parameter function does not lead to the fulfillment of the virial theorem relations for the polaron. So, the one-parameter function (5) is not sufficiently flexible. At the same time, for a two-parameter function (8) numerical verification showed that the known relations 1:3:4 of the virial theorem [21], which do not depend on the electronphonon interaction, are satisfied to within 7 significant digits. For the functional (13), the variation of which leads to a quadratic dependence on the parameter $\alpha$, the Pekar theorem for the strong coupling polaron 1:2:3:4 holds [19].

We verify the fulfillment of the virial theorem for the functional (20). The first term in Exp. (20) plays the role of kinetic energy for the limit $\alpha>>1$, the second term corresponds to the electron-phonon interaction $E_{\text {int }}$, the third one describes contribution of the phonon field $E_{\mathrm{ph}}$.

We introduce the following definitions in accord with [21]:

$F=E_{\text {kin }}+E_{\text {int }} / 2$ and $E_{\text {el }}=E_{\text {kin }}+E_{\text {int }}$.
As a result of numerical verification for the minimum of the functionals (14) and (20) in the asymptotics $\alpha>>1$, we obtain:

$$
\begin{aligned}
& E_{\text {kin }}=-F=0.2117827 \alpha^{4 / 3}, \\
& E_{\mathrm{el}} / 3=F=-0.2117827 \alpha^{4 / 3}, \\
& E_{\text {int }} / 4=F=-0.2117827 \alpha^{4 / 3}
\end{aligned}
$$

The relation $1: 2$, when $E_{\mathrm{ph}} / 2=-F$, is satisfied only in the case when the polaron energy is proportional to $\alpha^{2}$, which is possible in the strong coupling limit (Pekar theorem) [1].

As can be seen from Fig. 1a, the quadratic dependence of the polaron energy $E_{p s}^{\prime \prime} \approx-0.125 \alpha^{2}$ obtained by varying the approximate functional (13) with using the two-parameter function $f_{k}^{\prime}$ can serve as the upper limit of the polaron energy only in the region $\alpha \leq 7.5$. For the one-parameter $(N=1)$ function $f_{k}$ used in [8], the corresponding domain is somewhat expanded: $\alpha \leq 8.5$. Tulub [8] draws attention to the fact that in real crystals the maximum coupling constants can not exceed values $\alpha_{\max } \approx 8 \ldots 9$. The field of existence of the strongcoupling theory narrows to zero in fact. Fig. 1a can serve as a graphic illustration of this statement. The intersection point of the strong-coupling energy and the polaron energy obtained by varying the exact functionals (10) or (17) is a point near which minimization of the approximate functional (13) yields results that coincide with the minimization of the exact functionals. For smaller ones, it can serve as the upper limit of the polaron energy, at large it lowers the polaron energy in comparison with the value obtained by minimizing the exact functional.

\section{Conclusion}

The Gross-Tulub polaron functionals [6, 7] were determined only for the range of parameters, in which the function $D(s)$ is located in the first quarter of the complex plane. Therefore, these functionals can not be used to find the asymptotic dependences of the polaron energy for the region of extremely strong coupling.

Asymptotic dependences of the polaron energy in the region of ultimately large $\alpha$ are found using the functional obtained in [17]. This functional makes it possible to extend the range of admissible values of the parameters to the strong-coupling region in which the function $\operatorname{Re} D(s)$ can take negative values. The region of polaron existence on $\alpha$ can be conditionally divided into two parts: the region of continual polaron and region of extremely strong coupling for which the mathematical expressions obtained in the framework of the continuum theory are formal nature (a mathematical polaron). The region of existence of a continuum polaron for real crystals is limited by quantities 
$\alpha \approx 8 \ldots 10$. For this $\alpha$ and trial functions studied in this paper, the function $\operatorname{Re} D(s)>0$.

For the region of extremely strong coupling (the domain of existence of a mathematical polaron), the quantity $D(s)$ can be found both in the first and in the second quarter of the complex plane. In this region, the polaron energy tends to the asymptotic dependence defined by the expression (21). Approximate polaron functionals (14) and (20) minimized using the twoparameter test function $f_{k}^{\prime}$, satisfy the virial theorem $1: 3: 4$. That is valid for arbitrary electron-phonon interaction.

For $\alpha \approx 7.5 \ldots 8$, the function $\operatorname{Re} D(s)>0$, and the approximate Tulub functional (13) is a good approximation for the exact polaron functionals determined by the expressions (10) or (17). In this region, the polaron energy depends quadratically on $\alpha$ and the strong-coupling polaron virial theorem 1:2:3:4 holds.

The author is grateful to A.V. Tulub for numerous fruitful scientific advice and discussion of the results of work.

\section{References}

1. Pekar S.I. Research in Electron Theory of Crystals. Moscow, Publ. House Gostekhizdat, 1951 (in Russian).

2. Dykman M.I. and Rashba E.I. The roots of polaron theory. Physics Today. 2015. 68, No. 4. P. 10.

3. Alexandrov A.S., Devreese J.T. Advances in Polaron Physics. Springer, 2010.

4. Appel J. Polarons. In: Polarons. Part I. Ed. by A.Yu. Firsov. Moscow, Nauka, 1975.

5. Feynman R.P. Slow electrons in a polar crystal. Phys. Rev. 1955. 97, No. 3. P. 660-665.

6. Gross E.P. Small oscillation theory of the interaction of a particle and scalar field. Phys. Rev. 1955. 100, No. 6. P. 1571-1578.

7. Tulub A.V. Accounting the recoil in nonrelativistic quantum field theory. Vestnik Leningrad. gos. universiteta. Ser. 4. Fizika, Khimiya. 1960. 15, No. 22. P. 104-118 (in Russian).

8. Tulub A.V. Slow electrons in polar crystals. JETP. 1962. 14, No. 6. P. 1301-1307.
9. Lee T.D., Low F.E. and Pines D. The motion of slow electrons in a polar crystal. Phys. Rev. 1953. 90, No. 2. P. 297-302.

10. Tulub A.V. Comments on polaron-phonon scattering theory. Theoretical and Mathematical Physics. 2015. 185, No. 1. P. 1533-1546.

11. Lakhno V.D. Energy and critical ionic-bond parameter of a 3D large-radius bipolaron. JETF. 2010. 110, No. 5. P. 811-815.

12. Lakhno V.D. Translation-invariant bipolarons and the problem of high-temperature superconductivity. Solid State Communs. 2012. 152, No. 7. P. 621623.

13. Klimin S.N., Devreese J.T. Comments on "Translation-invariant bipolarons and the problem of high-temperature superconductivity". Solid State Communs. 2012. 152, No. 16. P. 1601-1603.

14. Lakhno V.D. On the cutoff parameter in the translation-invariant theory of the strong coupling polaron. Solid State Communs. 2012. 152, No. 19. P. 1855-1856.

15. Klimin S.N., Devreese J.T. Reply to "On the cutoff parameter in the translation-invariant theory of the strong coupling polaron". Solid State Communs. 2013. 153, No. 1. P. 58-59.

16. V.D. Lakhno, Pekar's ansatz and the strong coupling problem in polaron theory. PhysicsUspekhi. 2015. 58, No. 3. P. 295-308.

17. N.I. Kashirina, Gross-Tulub polaron functional in the region of intermediate and strong coupling. Semiconductor Physics, Quantum Electronics and Optoelectronics. 2017. 20, No. 3. P. 319-324.

18. Buimistrov V.M., Pekar S.I. The quantum states of particles coupled with arbitrary strength to a harmonically oscillating continuum. II. The case of translational symmetry. JETP. 1958. 6, No. 5. P. 977-980.

19. Kashirina N.I., Lakhno V.D., Tulub A.V. The virial theorem and the ground state problem in polaron theory. JETP. 2012. 114, No. 5. P. 867-869.

20. Miyake S.J. Strong-coupling limit of the polaron ground state. J. Phys. Soc. Jpn. 1975. 38, No. 1. P. 181-182.

21. Lemmens L.F., Devreese J.T. The 1:2:3:4 theorem and the ground state of free polarons. Solid State Communs. 1973. 12, No. 10. P. 1067-1069. 